\title{
Local delivery of recombinant adenovirus expressing hepatitis $B$ virus $X$ protein and interleukin-12 results in antitumor effects via inhibition of hepatoma cell growth and intervention of tumor microenvironment
}

\author{
HONG HE $^{1 *}$, PING FAN ${ }^{1 *}$, TAO YIN $^{1}$, QIAOLING CHEN ${ }^{1}$, HUASHAN SHI ${ }^{1}$, \\ SIJIA LIU ${ }^{1}$, HAOYU LI ${ }^{2}$, QIUPING JING ${ }^{1}$, YINGYING YAN ${ }^{1}$, HAILONG ZHANG ${ }^{1}$, \\ LI YANG $^{1}$, YANJUN WEN ${ }^{1}$, YUHUA LI ${ }^{1}$ and PING CHENG ${ }^{1}$ \\ ${ }^{1}$ State Key Laboratory of Biotherapy and Cancer Center, West China Hospital, West China Medical \\ School and ${ }^{2}$ School of Life Science, Sichuan University, Chengdu, Sichuan, P.R. China
}

Received January 22, 2012; Accepted March 26, 2012

DOI: $10.3892 /$ ijmm.2012.1027

\begin{abstract}
Hepatocellular carcinoma (HCC) is a typical hypervascular tumor. Our previous studies have demonstrated that hepatitis B virus $\mathrm{X}$ protein $(\mathrm{HBx})$ was able to inhibit the growth of HCC cells via inducing apoptosis and inhibiting tumor angiogenesis. Interleukin-12 (IL-12) is a disulfide-linked heterodimeric cytokine with potent immunostimulatory activity and anti-angiogenic properties. In this study, to further investigate the regulatory effect of IL-12 on HBx-mediated intervention of hepatoma microenvironment especially on intervention of neovessels and immune microenvironment, we constructed the recombinant adenovirus expressing HBx and mouse IL-12 named Ad-HBx-mIL-12. HBx-mIL-12 could effectively suppress tumor growth and induce apoptosis in vivo. Moreover, treatment with Ad-HBx-mIL-12 not only induced a massive accumulation of immune cells $\left(\mathrm{CD} 8^{+} \mathrm{T}\right.$ leukocytes, macrophages and dendritic cells) in tumors in situ, also apparently reduced the number of angiogenic blood vessels within tumor tissues. These results suggest that HBx-mIL-12 can not only induce cell cycle arrest and apoptosis in HCC cells, but also effectively shift the tumor microenvironment from pro-oncogenic to antitumor
\end{abstract}

Correspondence to: Dr Ping Cheng, State Key Laboratory of Biotherapy, West China Hospital, West China Medical School, Sichuan University, 1\# Keyuan Road 4, Gaopeng Street, High Technological Development Zone, Chengdu 610041, Sichuan, P.R. China

E-mail: ping.cheng@foxmail.com

${ }^{*}$ Contributed equally

Key words: hepatocellular carcinoma, hepatitis $\mathrm{B}$ virus $\mathrm{X}$ protein, mouse interleukin-12, tumor microenvironment, anti-angiogenic through recruitment of immune cells and inhibiting stromal cell growth, such as vascular endothelial cells.

\section{Introduction}

Hepatocellular carcinoma (HCC) is one of the most common and recurrent malignancies worldwide (1). Although the surgical techniques and various nonsurgical treatment modalities have improved, none of these therapies has significantly improved the extremely poor prognosis and the overall 5-year survival rate worldwide is only $2 \%$ (2). It is imperative that novel strategies against $\mathrm{HCC}$ are identified.

Hepatitis B virus X protein (HBx) is an HBV-encoded protein with multiple functions in transcription pathways, signal transduction, cell cycle progress and HBV replication (3). Studies have demonstrated that HBx could inhibit hepatocarcinoma cell growth via inducing apoptosis. In our previous studies, we showed that treatment with Ad-HBx intratumorally could not only induce apoptosis but also suppress angiogenesis (4).

HCC is a typical hypervascular tumor and tumor angiogenesis is required for both growth and metastasis of HCC $(5,6)$. Most patients show disease recurrence that rapidly progresses to the advanced stages with vascular invasion and multiple intrahepatic metastases (7). Our laboratory demonstrated that implantation of mesenchymal stem cells (MSCs) that are genetically modified with adenovirus vector encoding interleukin-12 (IL-12) could significantly suppress HCC tumor growth in vivo via inhibiting tumor angiogenesis (8).

Not only neovascular endothelial cells that are critical components of the tumor microenvironment, but also the immunocytes in the tumor stroma can affect tumor prognosis through participating innate and adaptive immunity (9). Many studies showed that $\mathrm{HBx}$ could elicit cytotoxic $\mathrm{T}$ lymphocyte (CTL) antitumor immune responses. Vaccines based on HBx full-length sequence or specific epitopes could trigger significant immune reactions (10-13). Our previous studies showed that immunotherapy with Ad-HBx vaccine induced a massive accumulation of $\mathrm{CD} 8^{+} \mathrm{T}$ cells at the tumor site and accord- 
ingly elicit the host CTL response against HBV-associated HCC (11). Intratumoral injections with Ad-HBx induced not only apoptosis of HCC cells but also lymphocytes infiltration in the tumor stroma.

IL-12 as a heterodimeric cytokine, has potent anti-tumor activity and anti-metastatic effect. A large number of animal experiments demonstrated that IL-12 could inhibit tumor angiogenesis and participate in immunoregulation $(14,15)$. IL-12 significantly promoted the NK cell and T cell proliferation, induced the production of IFN- $\gamma$ and stimulated naive $\mathrm{CD} 4^{+} \mathrm{T}$ cells to differentiate toward the Th1 phenotype (16-18). Recently, combination IL-12 with other treatment options was proved to be a better anti-tumor effect with lower side-effect (19-21).

In this study, to further investigate the regulatory effect of IL-12 on HBx mediated intervention of hepatoma microenvironment especially on intervention of neovessels and immune microenvironment, we constructed the recombinant adenovirus carrying HBx and mIL-12 named Ad-HBx-mIL-12.

\section{Materials and methods}

Cell lines and adenovirus preparation. Hepa1-6 (mouse HCC) cell line, which was purchased from the American Type Culture Collection, was cultured in DMEM medium (Gibco-BRL) with $10 \%$ fetal bovine serum (FBS; Gibco-BRL) and $10 \mu \mathrm{g} / \mathrm{ml}$ gentamicin sulfate at $37^{\circ} \mathrm{C}$ in $5 \% \mathrm{CO}_{2}$. Ad-HBx-mIL-12 is an E1, E3-deleted recombinant adenoviruse (rAd) expressing the HBx and murine IL-12. Ad-HBx, Ad-mIL-12 and Ad-null are E1, E3-deleted rAd expressing the HBx, murine IL-12 and no transgene, respectively. All recombinant adenoviruses were constructed and conserved by our laboratory.

Hepal-6 cell cycle and apoptosis assay in vitro. Hepa1-6 cells were plated into 6 -well plates at $5.0 \times 10^{5}$ cells/well, overnight. Then, cells were left untreated or infected with Ad-null, Ad-mIL-12, Ad-HBx and Ad-HBx-mIL-12. After $48 \mathrm{~h}$, cell cycle profiles (MOI 10) and apoptosis (MOI 30) were analyzed by flow cytometer (Beckman Coulter) after propidium iodide (PI) staining or Annexin V-phycoerythrin/ PI staining, respectively.

In vivo studies. Female syngeneic C57BL/6 mice, 6-8 weeks old, were obtained from Beijing Hua Fu Kang biological technology company and maintained in pathogen-free conditions. All procedures were reviewed and approved by the Institute Animal Care and Use Committee. Mice were challenged subcutaneously with $5 \times 10^{6}$ Hepa1-6 cells in the right flank. When tumors had reached the desired size $\left(100 \mathrm{~mm}^{3}\right)$, mice were grouped randomly $(\mathrm{n}=10)$ and treatment was initiated. Ad-null, Ad-HBx, Ad-mIL-12 and Ad-HBx-mIL-12, diluted in $100 \mu 1$ sterile 1x PBS (Sigma-Aldrich), were administered 4 times, 5 days apart, intratumorally, at the dose of $10^{8}$ plaqueforming units (pfu). Tumor dimensions were measured with calipers every 3 days and tumor volumes were calculated according to the formula: $\mathrm{V}=$ length $\mathrm{x}$ width $\mathrm{x} \pi / 6$.

Tumor microenvironment analysis. For FACS analysis, we prepared single-cell suspensions of tumors from untreated or treated mice. Briefly, tumors were minced using a sterile razor blade and digested with collagenase I. For extracellular staining of immune markers, $1 \times 10^{6}$ of freshly prepared cells were stained with different combinations of fluorochromecoupled antibodies to CD4, CD8, CD11b, CD11c, F4/80 and Gr1 (BD Biosciences). Fluorescence data were collected on FACScalibur and analyzed using cell quest software (BD Biosciences).

Immunohistochemistry. Three days after the completion of treatment, the mice were sacrificed for histological analysis. The tissues were fixed in $4 \%$ paraformaldehyde. Primary tumors were embedded in paraffin and cut into 3-5 $\mu \mathrm{m}$ sections. The apoptotic cells within the tumor sections were evaluated by TUNEL staining, using DeadEnd ${ }^{\mathrm{TM}}$ Fluorometric TUNEL System (Promega). Apoptosis index was determined by counting the number of apoptotic cells and dividing by the total number of cells in the field (5 high power fields/slide). Frozen tumor tissues were stained for the quantification of microvessel density (MVD) using a monoclonal rabbit anti-mouse CD31phcoerythrin conjugate (Santa Cruz Biotechnology, Inc.).

Toxicity evaluation. To investigate potential side effects or toxicity in the treated mice, they were observed continuously for relevant indexes such as weight loss, ruffled fur, diarrhea, anorexia, cachexia, skin ulceration or toxic deaths. After fixing in $4 \%$ neutral buffered formalin solution more than $24 \mathrm{~h}$, the tissues of heart, liver, spleen, lung, kidney and brain were embedded in paraffin. Slices of 3-5 $\mathrm{mm}$ were stained with hematoxylin and eosin (H\&E) and observed in double blinded manner.

Statistical analysis. SPSS 17.0 was used for statistical analysis. Data were expressed as the mean $\pm \mathrm{SE}$. The statistical analysis in all the experiments was performed using one-way analysis of variance (ANOVA) or unpaired Student's t-test. P-values $<0.05$ were considered significant.

\section{Results}

HBx-mIL-12 induces HCC cells to cell cycle arrest and apoptosis in vitro. Hepa1-6 cells were left untreated or infected by Ad-null, Ad-mIL-12, Ad-HBx and Ad-HBx-mIL-12. After $48 \mathrm{~h}$, cell cycle profiles (MOI 10) and apoptosis (MOI 30) were analyzed by flow cytometer. Results showed that Ad-HBx and Ad-HBx-mIL-12 induced a significant accumulation of cells in G2/M phase accompanied by a decrease in the percentage of cells in G0/G1 phase and S phase (Fig. 1A). The percentages of Ad-HBx and Ad-HBx-mIL-12 infected Hepa1-6 cells in G2/M phase were $22.25 \pm 1.8 \%$ and $26.15 \pm 2.1 \%$, respectively, and higher than those of untreated, Ad-null and Ad-mIL-12 infected cells $(\mathrm{P}<0.01)$ (Fig. 1B). With increasing MOI, flow cytometric analysis revealed that Ad-HBx and Ad-HBx-mIL-12 infected cells underwent significant apoptosis (Fig. 1C). The percentages of apoptosis in Ad-HBx and Ad-HBx-mIL-12 infected groups were $63.17 \pm 5.2 \%$ and $61.68 \pm 3.1 \%$, respectively, and higher than those of untreated, Ad-null and Ad-mIL-12 infected groups $(\mathrm{P}<0.01)$ (Fig. 1D). According to our flow cytometric analysis, the cell cycle arrest and apoptosis in Ad-HBx and Ad-HBx-mIL-12 infected groups have no significant deviation. These results indicate that HBx could induce 

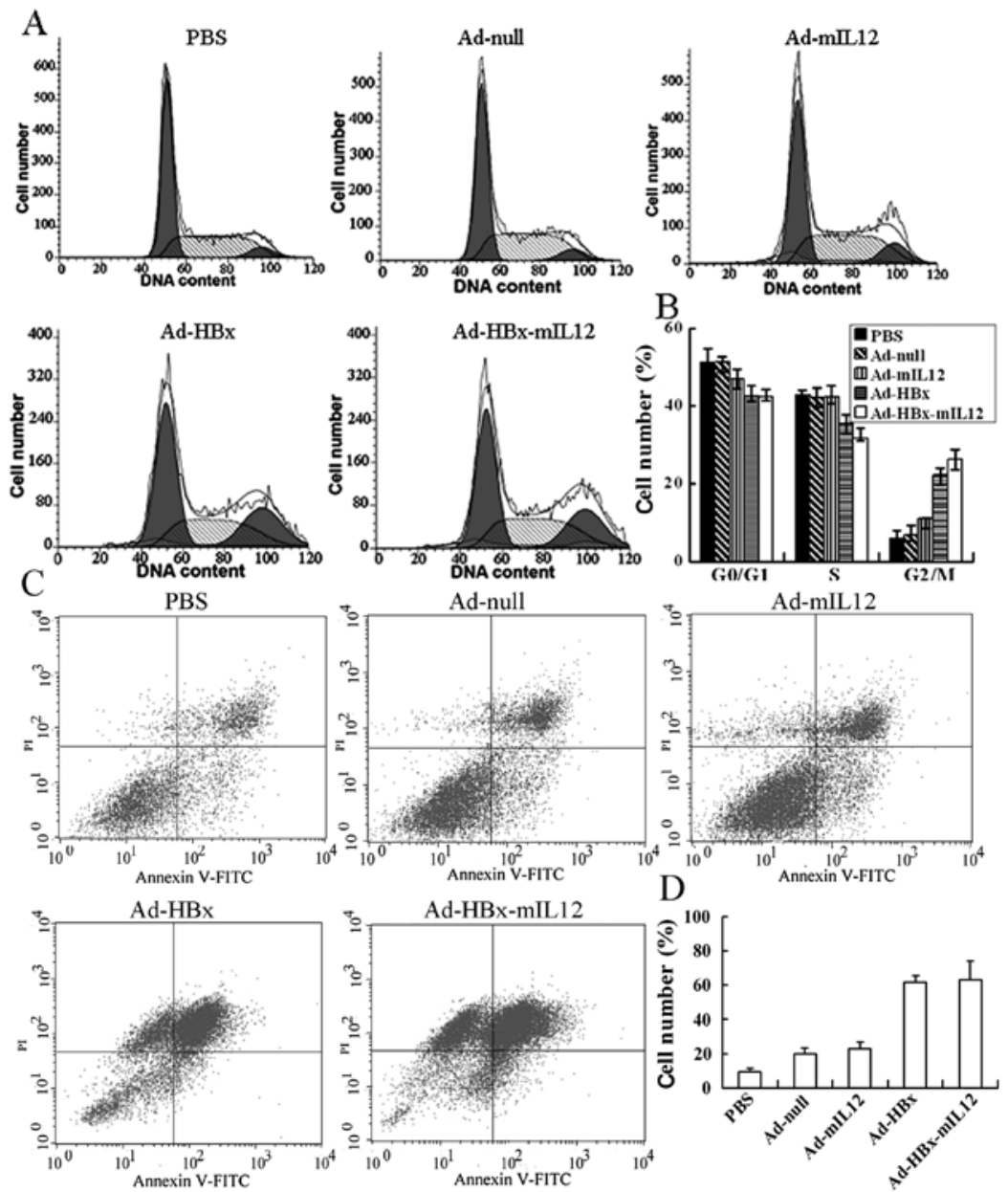

Figure 1. Effects of HBx-mIL-12 and HBx on cell cycle distribution and apoptosis in HCC cells. The cell cycle arrest and apoptosis in Ad-HBx and Ad-HBx-mIL-12 infected groups have no significant deviation. These results indicate that HBx could induces HCC cells to G2/M arrest and apoptosis, whereas mIL-12 have no function in inducing cell cycle arrest and apoptosis of HCC cells in vitro. (A) HBx induced a significant accumulation of cells in G2/M phase. (B) Quantification of cell cycle distribution. (C) Apoptosis was significantly increased in Ad-HBx-mIL-12 and Ad-HBx groups compared with other groups . (D) Quantification of the percentages of apoptosis. The quantification of cell cycle distribution and percentages of apoptosis was performed using CellQuest software. Data from 3 independent experiments are shown as the mean \pm SD.
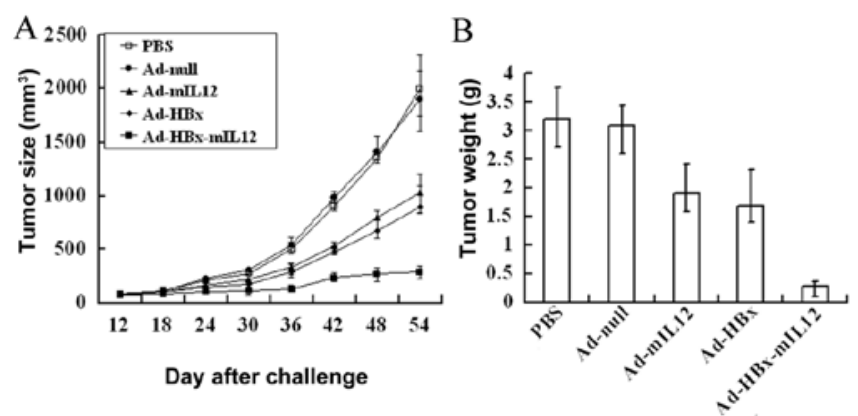

Figure 2. Antitumor efficacy of HBx-mIL-12 in vivo. The mice were treated with corresponding recombinant adenoviruses at the dose of $10^{8}$ plaqueforming units intratumorally. (A) Tumor sizes on each mouse were monitored every 3 days and (B) tumor weights were measured. HBx-mIL-12 can significantly suppress tumor growth $(\mathrm{P}<0.01)$. Point, average tumor volume or weights; bar \pm SD.

HCC cells to G2/M arrest and apoptosis, whereas mIL-12 has no function in inducing cell cycle arrest and apoptosis of HCC cells in vitro.
HBx-mIL-12 inhibits the growth of HCC in vivo. A murine hepatic carcinoma model was established to investigate the anti-tumor effect of Ad-HBx-mIL-12 in vivo. When tumors had reached the desired size $\left(100 \mathrm{~mm}^{3}\right)$, Ad-null, Ad-HBx, Ad-mIL-12, Ad-HBx-mIL-12 or PBS was administered intratumorally. Alterations in tumor growth were monitored every 3 days (Fig. 2A). The Ad-HBx-mIL-12 group exhibited effective inhibition of tumor growth, compared to the other groups $(\mathrm{P}<0.01)$. On Day 54 after the Hepa1-6 cells were challenged, the mice were sacrificed and the tumor weights were measured (Fig. 2B). We observed that treatment with Ad-HBx-mIL-12 resulted in average tumor weight reductions of $85.6 \pm 4.9 \%$, $85.1 \pm 2.8 \%, 75.8 \pm 6.1 \%$ and $72.8 \pm 5.6 \%$ compared to PBS, Ad-null, Ad-mIL-12 and Ad-HBx, respectively $(\mathrm{P}<0.01)$. These results indicate that Ad-HBx-mIL-12 can effectively suppress tumor growth.

HBx-mIL-12 induces apoptosis of HCC cells in vivo. In order to estimate the apoptosis in tumor tissues, TUNEL staining was applied to the tumor sections. We observed more apoptotic cells in the tumor sections of mice treated with Ad-HBx-mIL-12 than with PBS, Ad-null, Ad-mIL-12 and 


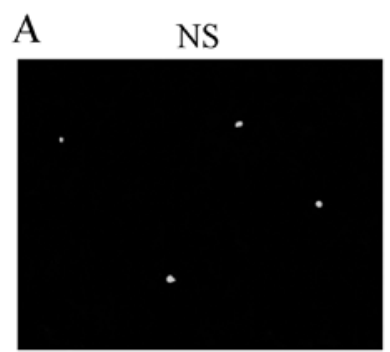

Ad-HBx

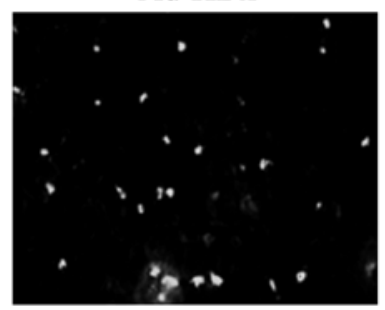

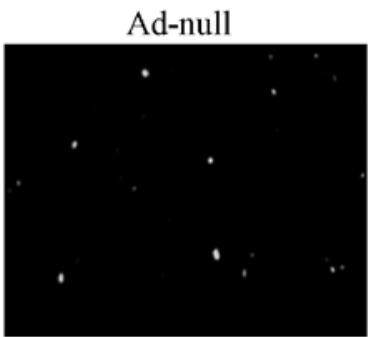

Ad-HBx-mIL12

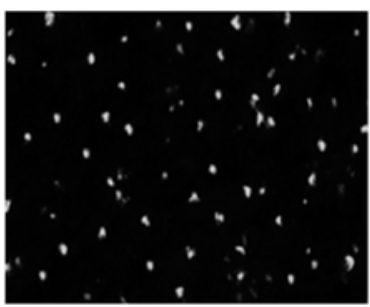

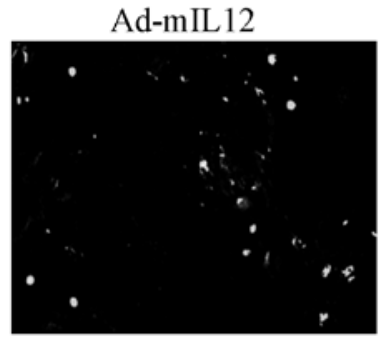

B

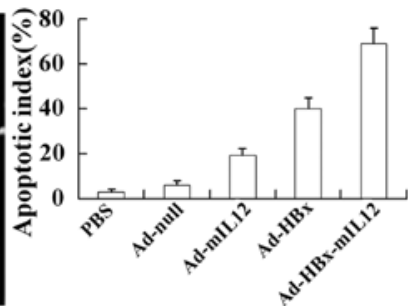

Figure 3. Detection of apoptosis by TUNEL staining of tumor tissues. On Day 54, tumor tissues were sectioned and stained with FITC-dUTP. The percentage of apoptosis was determined by counting the number of apoptotic cells and dividing by the total number of cells in the field. (A) TUNEL staining in each group (original magnification, x200). (B) Apoptotic index of Ad-HBx-mIL-12 treated tumors was higher than that of other groups $(\mathrm{P}<0.05)$. Columns, mean of apoptotic index; bar $\pm \mathrm{SD}$.

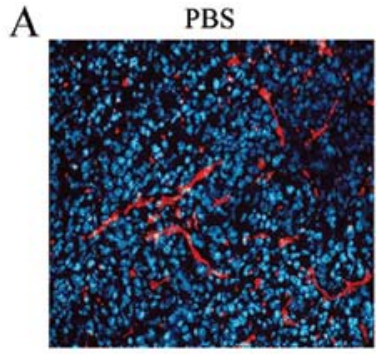

Ad-HBx

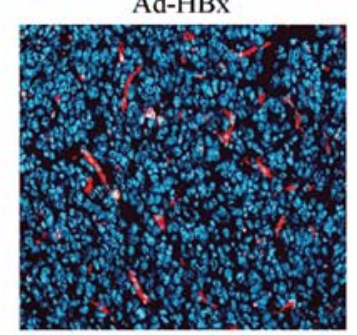

Ad-null

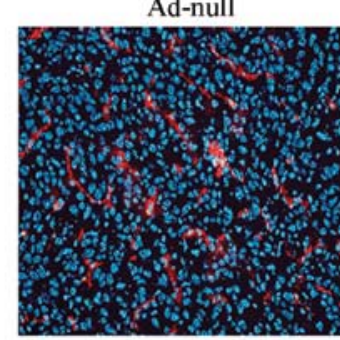

Ad-HBx-mIL12

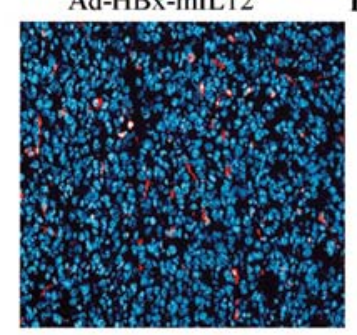

Ad-mIL12

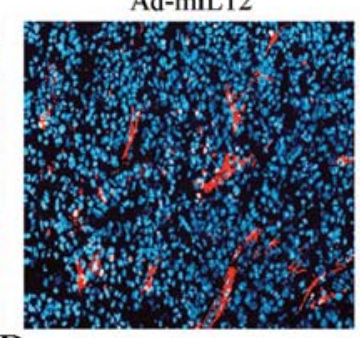

B

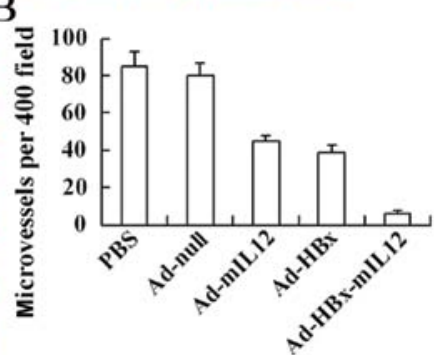

Figure 4. Inhibition of angiogenesis within tumor tissue stained by CD31. On Day 54, tumor tissues were sectioned and stained with CD31. Vascular density was estimated by counting the number of microvessels/high power field. (A) CD31 staining of microvessels in each group (original magnification, x400). (B) Treatment with Ad-HBx-mIL-12 reduced the number of microvessel compared with other groups $(\mathrm{P}<0.05)$. Columns, mean of microvessel/high-power field; bar \pm SD.

Ad-HBx (Fig. 3A). The apoptosis index also showed that the Ad-HBx-mIL-12 displayed the highest indices among all the groups (Fig. 3B).

HBx-mIL-12 inhibits vascular endothelial cell growth in vivo. We further investigated whether Ad-HBx-mIL-12 also had inhibitory effects on tumor-stromal cells besides HCC cells within treated tumor tissue. Angiogenic blood vessels within tumor tissue were detected by CD31 immunohistochemistry. The frozen sections were stained for angiogenic blood vessels using a monoclonal rabbit anti-mouse CD-31-phcoerythrin conjugate and counterstained with DAPI (Fig. 4A). Tumors of the control groups (including PBS, Ad-null, Ad-mIL-12 and Ad-HBx groups) showed larger microvessel count than those of Ad-HBx-mIL-12 group (Fig. 4B).

HBx-mIL-12 causes a significant increase in tumor-infiltrating $C D 8^{+} T$-cell. To further assess the role of immune modulation in the observed antitumor effects mediated by Ad-HBx-mIL-12 treatment, we analyzed the modulation of tumor microenvironment. The ratio of $\mathrm{T}$ cells within the tumor microenvironment is considered indicative of the effect of adaptive immune responses on tumor progression and metastasis $(19,20)$. We investigated the subsets of tumor-infil- 

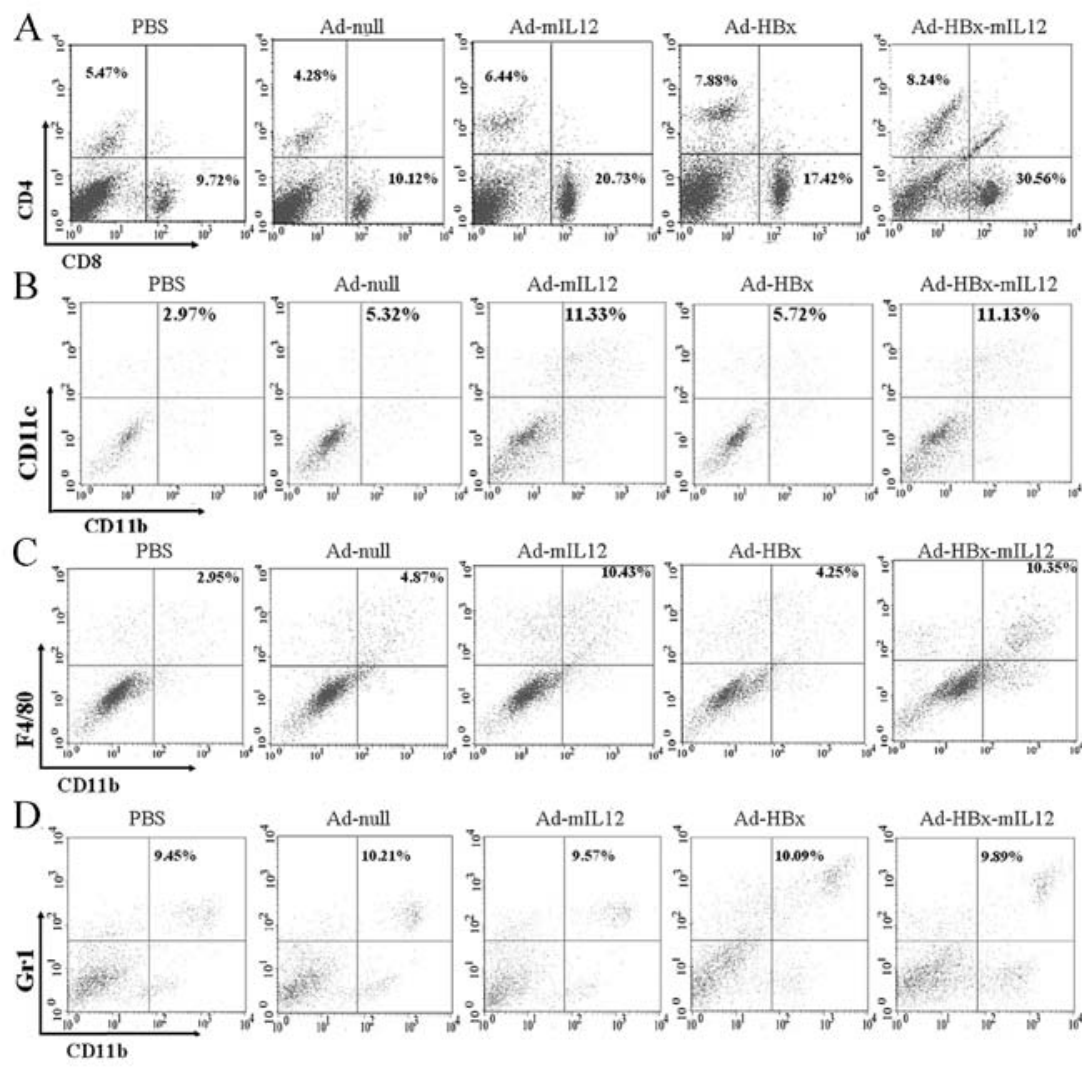

Figure 5. Effects of in vivo Ad-HBx-mIL-12 treatment on tumor-infiltrating cell populations within HCC tumor microenvironment.(A-D) Single-cell suspensions prepared from tumors pooled from three mice/group were analyzed by flow cytometry for the presence of CD4, CD8, CD11b, CD11c, F4/80 and Gr1 antibodies. (A) Infiltration of CD4 and CD8 $\mathrm{T}$ cells in the tumor region. (B,C) The increases in tumor-infiltrating DC (CD11b $\left.{ }^{+} \mathrm{CD} 11 \mathrm{c}^{+}\right)$and macrophage

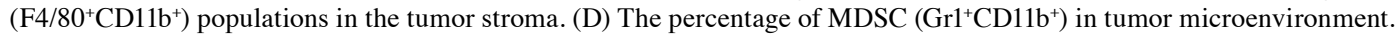

trating T-cell populations by staining with anti-CD4-PE and CD8-fluorescein isothiocyanate. Although Ad-HBx-mIL-12 treatment did not induce significant change in the ratio of $\mathrm{CD} 4^{+} \mathrm{T}$-cells, we observed an increase in the infiltration of total $\mathrm{CD}^{+} \mathrm{T}$ cells in the tumor stroma, as shown by flow cytometric analysis (Fig. 5A). These effects probably result from $\mathrm{HBx}$ and mIL-12 mediating host CTL response.

HBx-mIL-12 induces an increase in tumor-infiltrating DCs and macrophages. It has been documented that tumor-infiltrating dendritic cells (DC) and macrophages are potentially able to induce an antitumor innate and adaptive immune response

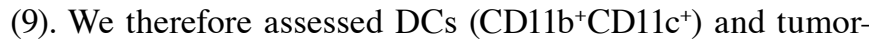
infiltrating macrophage $\left(\mathrm{F} 4 / 80^{+} \mathrm{CD} 11 \mathrm{~b}^{+}\right)$populations by flow cytometry, which showed an increase in DCs and macrophages in tumors after local treatment with Ad-HBx-mIL-12 and Ad-mIL-12 (Fig. 5B and C). However, the increased ratios of DCs and macrophages in Ad-mIL-12 and Ad-HBx-mIL-12 treated tumors have no significant deviation. The numbers of tumor-infiltrating DCs and macrophages in Ad-HBx and Ad-null treated tumors have no significant increase compared with that in PBS group. These results indicate that the increased ratios of tumor-infiltrating DCs and macrophages potentially result from IL-12 mediating the immune responses.

HBx-mIL-12 has no impact on the percentage of MDSCs in tumor microenvironment. Myeloid-derived suppressor cells (MDSCs) in the tumor microenvironment produce growth factors and angiogenic factors critical for tumor progression $(20,21)$. Thus, a large number of MDSCs in tumor microenvironment have probably suppressive actions in the treatment of tumor. We therefore assessed MDSCs $\left(\mathrm{Grl}^{+} \mathrm{CD} 1 \mathrm{lb}^{+}\right)$populations by flow cytometry, which showed the percentage of MDSCs in Ad-HBx-mIL-12 treated tumors did not have significant changes compared with control groups (including PBS, Ad-null, Ad-mIL-12 and Ad-HBx groups) (Fig. 5D). These result indicate that Ad-HBx-mIL-12 have no effect on decreasing the percentage of MDSCs in tumor microenvironment.

\section{Discussion}

In the present study, we demonstrated that HBx-mIL-12 has more anti-tumor effect when compared with HBx or IL-12, as shown in our previous studies (4). In vitro, we found that Hepa1-6 cells infected with Ad-HBx and Ad-HBx-mIL-12 underwent G2/M arrest and apoptosis. However IL-12 did not affect the function of HBx. In vivo, we established a mouse tumor model and performed intratumoral injection of Ad-HBx-mIL-12. We found that although IL-12 did not affect the function of $\mathrm{HBx}$ in vitro, combination of IL-12 with HBx induced higher apoptotic effect than HBx or IL-12 in vivo. Combination of IL-12 with $\mathrm{HBx}$ led to an almost complete eradication of HCC. The anti-tumor mechanisms of HBx-mIL-12 are due not only to induction of cell cycle arrest and apoptosis in HCC cells, but also effectively shift the tumor microenvironment from pro-oncogenic to anti-tumor. 
Although tumors are capable of autonomous growth, their progression is highly influenced by their stromal component (22). T cells, tumor-infiltrating DC, tumor-associated macrophages and myeloid cells are critical components of the tumor microenvironment and the cells may exert either inhibiting or promoting effects on tumor growth (23) through participating innate and adaptive immunity $(21,24,25)$. The ratio of $\mathrm{T}$ cells within the tumor microenvironment is considered indicative of the effect of adaptive immune responses on tumor progression and metastasis (19). Abundant $\mathrm{CD}^{+} \mathrm{T}$-cell infiltration in the tumor stroma is associated with a favorable course of malignancy in cancer (26-29). Previous studies have demonstrated that $\mathrm{HBx}$ could induce infiltration of $\mathrm{CD}^{+}$ $\mathrm{T}$ lymphocytes in $\mathrm{HBx}$-positive tumors (11). In addition, IL-12 can induce an efficient antitumor $\mathrm{CD} 8^{+} \mathrm{CTL}$ response which is regarded as the major mediators of the natural host response against developing tumors $(30,31)$. Thus, there is a hypothesis that IL-12 can enhance CD8 ${ }^{+}$CTL response elicited by HBx. In our study, increased $\mathrm{T}$ cell infiltration, especially $\mathrm{CD}^{+}$cells, can be detected in tumor tissue of mice treated with Ad-HBx-mIL-12 compared to control groups. This observation is consistent with other studies, indicating that a higher infiltration $\mathrm{CD}^{+}$ratio within the tumor microenvironment has been correlated with a favorable response to the treatment, as compared with unchanged ratios in non-responders.

Many studies have definitively shown that recruitment of various antigen-presenting cells, such as dendritic cells (DCs) and macrophages, within tumors can initiate a cascade of innate, adaptive or humoral immune responses against growing tumors $(20,32,33)$. Our study showed an increase in the infiltration of DCs and macrophages in the tumor stroma, after local treatment with Ad-HBx-mIL-12 and Ad-mIL-12. However, the increased ratios of DCs and macrophages in Ad-mIL-12 and Ad-HBx-mIL-12 treated tumors have no significant deviation. These results indicate that the increased ratios of tumor-infiltrating DCs and macrophages potentially result from IL-12 mediating the immunological reaction. Indeed, our findings support the view that IL-12, a key cytokine in cancer immunoprevention, seems to induce infiltration of activated DCs and macrophages to tumor and different organs $(34,35)$.

In our study, treatment with Ad-HBx-mIL-12 reduced the number of angiogenic blood vessels within tumor tissues compared with control group. Studies have shown that MDSCs in the tumor microenvironment produce growth factors and angiogenic factors critical for tumor progression (21). However, the percentage of MDSCs in treated tumors did not decrease compared with control groups. The anti-angiogenic property of IL-12 was well documented in different in vitro and in vivo studies $(36,37)$. In our previous study, treatment with Ad-HBx apparently reduced the number of angiogenic blood vessels within HCC tumor tissue (4). Thus, the mechanism of the anti-angiogenic effect of Ad-HBx-mIL-12 in the present study may result from IL-12 and HBx mediated the anti-angiogenic effects.

In our study, treatment with Ad-HBx-mIL-12 not only induced cell cycle arrest and apoptosis in HCC cells, also induced a massive accumulation of immune cells $\left(\mathrm{CD}^{+} \mathrm{T}\right.$ leukocytes, macrophages and DC) and significant reduc- tion of vascular endothelial cell growth tumors in situ. HBx-mIL-12 could shift the tumor microenvironment from pro-oncogenic to antitumor not only through recruitment immune cells but also inhibiting stromal cell growth. Collectively, our data in the present study suggest that local delivery of Ad-HBx-mIL-12 could result in antitumor effects via inhibition of hepatoma cell growth and intervention of tumor microenvironment.

\section{Acknowledgements}

This study is supported by the National Natural Science Foundation of China (Grant no. 81101728), the National Science and Technology Major Projects of New Drugs (Grant no. 2012ZX09103301-036), the National Science and Technology Major Project for Infectious Diseases Control (Grant no. 2012ZX10002014-004) and the Research Fund for the Doctoral Program of Higher Education of China (Grant no. 20110181120086).

\section{References}

1. Beasley RP: Rocks along the road to the control of HBV and HCC. Ann Epidemiol 19: 231-234, 2009.

2. Okuda K: Hepatocellular carcinoma. J Hepatol 32: 225-237, 2000.

3. Tang H, Oishi N, Kaneko S and Murakami S: Molecular functions and biological roles of hepatitis B virus x protein. Cancer Sci 97: 977-983, 2006.

4. Cheng P, Li Y, Yang L, et al: Hepatitis B virus X protein (HBx) induces $\mathrm{G} 2 / \mathrm{M}$ arrest and apoptosis through sustained activation of cyclin B1-CDK1 kinase. Oncol Rep 22: 1101-1107, 2009.

5. Tian T, Nan KJ, Wang SH, et al: PTEN regulates angiogenesis and VEGF expression through phosphatase-dependent and -independent mechanisms in HepG2 cells. Carcinogenesis 31: 1211-1219, 2010.

6. Fernandez M, Semela D, Bruix J, Colle I, Pinzani M and Bosch J: Angiogenesis in liver disease. J Hepatol 50: 604-620, 2009.

7. Liu L, Cao Y, Chen C, et al: Sorafenib blocks the RAF/MEK/ ERK pathway, inhibits tumor angiogenesis, and induces tumor cell apoptosis in hepatocellular carcinoma model PLC/PRF/5. Cancer Res 66: 11851-11858, 2006.

8. Chen X, Lin X, Zhao J, et al: A tumor-selective biotherapy with prolonged impact on established metastases based on cytokine gene-engineered MSCs. Mol Ther 16: 749-756, 2008.

9. Guiducci C, Vicari AP, Sangaletti S, Trinchieri G and Colombo MP: Redirecting in vivo elicited tumor infiltrating macrophages and dendritic cells towards tumor rejection. Cancer Res 65: 3437-3446, 2005

10. Chun E, Lee J, Cheong HS and Lee KY: Tumor eradication by hepatitis $\mathrm{B}$ virus $\mathrm{X}$ antigen-specific $\mathrm{CD} 8^{+} \mathrm{T}$ cells in xenografted nude mice. J Immunol 170: 1183-1190, 2003.

11. Li Y, Cheng P, Wen Y, et al: T lymphocyte responses against hepatitis B virus-related hepatocellular carcinoma induced by adenovirus vaccine encoding HBx. Int J Mol Med 26: 869-876, 2010.

12. Ding FX, Wang F, Lu YM, et al: Multiepitope peptide-loaded virus-like particles as a vaccine against hepatitis $B$ virus-related hepatocellular carcinoma. Hepatology 49: 1492-1502, 2009.

13. Wang YJ, Hou Y, Huang H, Liu GR, White AP and Liu SL: Two oral HBx vaccines delivered by live attenuated Salmonella: both eliciting effective anti-tumor immunity. Cancer Lett 263: 67-76, 2008.

14. Hombach A, Heuser C and Abken H: Simultaneous targeting of IL2 and IL12 to Hodgkin's lymphoma cells enhances activation of resting NK cells and tumor cell lysis. Int J Cancer 115: 241-247, 2005

15. Halin C, Rondini S, Nilsson F, et al: Enhancement of the antitumor activity of interleukin-12 by targeted delivery to neovasculature. Nat Biotechnol 20: 264-269, 2002.

16. Peng LS, Penichet ML and Morrison SL: A single-chain IL-12 IgG3 antibody fusion protein retains antibody specificity and IL-12 bioactivity and demonstrates antitumor activity. J Immunol 163: 250-258, 1999. 
17. Hendrzak JA and Brunda MJ: Antitumor and antimetastatic activity of interleukin-12. Curr Top Microbiol Immunol 213: 65-83, 1996.

18. Trinchieri G: Immunobiology of interleukin-12. Immunol Res 17: 269-278, 1998

19. Nasu Y, Bangma $\mathrm{CH}$, Hull GW, et al: Combination gene therapy with adenoviral vector-mediated HSV-tk ${ }^{+}$GCV and IL-12 in an orthotopic mouse model for prostate cancer. Prostate Cancer Prostatic Dis 4: 44-51, 2001.

20. Couglin CM, Salhang KE, Wysocka M, et al: Interleukin-12 and interleukin-18 synergistically induce murine tumor regression which involves inhibition of angiogenesis. J Clin Invest 101: 1441-1452, 1998

21. Pützer BM, Hitt M, Muller WJ, et al: Interleukin 12 and B7-1 co-stimulatory molecule expressed by an adenovirus vector act synergistically to facilitate tumor regression. Proc Natl Acad Sci USA 94: 10889-10895, 1997.

22. Bui JD, Uppaluri R, Hsieh CS and Schreiber RD: Comparative analysis of regulatory and effector $\mathrm{T}$ cells in progressively growing versus rejecting tumors of similar origins. Cancer Res 66: 7301-7309, 2006.

23. Kortylewski M, Swiderski P, Herrmann A, et al: In vivo delivery of siRNA to immune cells by conjugation to a TLR9 agonist enhances antitumor immune responses. Nat Biotechnol 27: 925-932, 2009.

24. Kujawski M, Kortylewski M, Lee H, Herrmann A, Kay H and $\mathrm{Yu} \mathrm{H}$ : Stat 3 mediates myeloid cell-dependent tumor angiogenesis in mice. J Clin Invest 118: 3367-3377, 2008.

25. Coussens LM and Werb Z: Inflammation and cancer. Nature 420 860-867, 2002

26. Mantovani A, Sozzani S, Locati M, Allavena P and Sica A Macrophage polarization: tumor-associated macrophages as a paradigm for polarized M2 mononuclear phagocytes. Trends Immunol 23: 549-555, 2002.

27. Rodriguez PC, Ernstoff MS, Hernandez C, et al: Arginase I-producing myeloid-derived suppressor cells in renal cell carcinoma are a subpopulation of activated granulocytes. Cancer Res 69: 1553-1560, 2009.

28. Norian LA, Rodriguez PC, O'Mara LA, et al: Tumor-infiltrating regulatory dendritic cells inhibit $\mathrm{CD} 8^{+} \mathrm{T}$ cell function via L-arginine metabolism. Cancer Res 69: 3086-3094, 2009.
29. Piersma SJ, Jordanova ES, van Poelgeest MI, et al: High number of intraepithelial CD $8^{+}$tumor-infiltrating lymphocytes is associated with the absence of lymph node metastases in patients with large early-stage cervical cancer. Cancer Res 67: 354-361, 2007.

30. Menon AG, Fleuren GJ, Alphenaar EA, et al: A basal membranelike structure surrounding tumour nodules may prevent intraepithelial leucocyte infiltration in colorectal cancer. Cancer Immunol Immunother 52: 121-126, 2003.

31. Sato E, Olson $\mathrm{SH}$, Ahn J, et al: Intraepithelial $\mathrm{CD}^{+}$tumorinfiltrating lymphocytes and a high $\mathrm{CD}^{+} /$regulatory $\mathrm{T}$ cell ratio are associated with favorable prognosis in ovarian cancer. Proc Natl Acad Sci USA 102: 18538-18543, 2005.

32. Zhang L, Conejo-Garcia JR, Katsaros D, et al: Intratumoral T cells, recurrence, and survival in epithelial ovarian cancer. $N$ Engl J Med 348: 203-213, 2003.

33. Simma O, Zebedin E, Neugebauer N, et al: Identification of an indispensable role for tyrosine kinase 2 in CTL-mediated tumor surveillance. Cancer Res 69: 203-211, 2009.

34. Torrero MN, Xia X, Henk W, Yu S and Li S: Stat1 deficiency in the host enhances interleukin-12-mediated tumor regression. Cancer Res 66: 4461-4467, 2006.

35. Kanzler H, Barrat FJ, Hessel EM and Coffman RL: Therapeutic targeting of innate immunity with Toll-like receptor agonists and antagonists. Nat Med 13: 552-559, 2007.

36. Barchet W, Wimmenauer V, Schlee M and Hartmann G Accessing the therapeutic potential of immunostimulatory nucleic acids. Curr Opin Immunol 20: 389-395, 2008.

37. Tannenbaum CS, Wicker N, Armstrong D, et al: Cytokine and chemokine expression in tumors of mice receiving systemic therapy with IL-12. J Immunol 156: 693-699, 1996.

38. Adris S, Chuluyan E, Bravo A, et al: Mice vaccination with interleukin 12-transduced colon cancer cells potentiates rejection of syngeneic non-organ-related tumor cells. Cancer Res 60: 6696-6703, 2000.

39. Nastala CL, Edington HD, McKinney TG, et al: Recombinant IL-12 administration induces tumor regression in association with IFN-gamma production. J Immunol 153: 1697-1706, 1994.

40. Luster AD and Leder P: IP-10, a -C-X-C- chemokine, elicits a potent thymus-dependent antitumor response in vivo. J Exp Med 178: 1057-1065, 1993. 